\title{
In Vitro Assessment of Dermal Absorption for Flame Retardant Textile Finishing Chemicals
}

\author{
Martí M*, Alonso C, Manich AM and Coderch L \\ Institute of Advanced Chemistry of Catalonia, IQAC-CSIC, Barcelona, Spain
}

*Corresponding author: Meritxell Martí, Institute of Advanced Chemistry of Catalonia, IQAC-CSIC, Jordi Girona 18-26, 08034 Barcelona, Spain

Received Date: June 11, 2020

Published Date: June 23, 2020

\begin{abstract}
Flame Retardants are a group of anthropogenic environmental contaminants used in textile finishing's. Currently, the largest marked group of FRs is brominated FR considered toxic, persistent and bio accumulative. Non-halogenated alternatives are a possible solution, but there is a lack of knowledge concerning environmental impact and health risks.

The aim of this study is focused on dermal absorption of FR in order to calculate the Margin of security for textile fabrics treated with flame retardant finishing's. FR based on sulfamate ammonium were used applied onto cotton and polyester fabrics. Both have been demonstrated that they are dermal safety for human at the conditions used.
\end{abstract}

Keywords: Flame retardants; Percutaneous absorption; MoS; Sulfamate ammonium; Cotton; Polyester

Abbreviations: FR: Flame Retardant; OECD: Organization for Economic Cooperation and Development: MoS: Margin of Security; SED: Systemic Exposure Dose; NOAEL: Non-Observed Adverse Effect Level

\section{Introduction}

Flame retardant finishing's could be considered somehow dangerous to human health and to environment, although much progress has been made in the industry to reach its complete replacement.

FRs are compounds that inhibit or delay combustion. The term "flame retardant" does not refer to a specific class of chemical, rather describes the function of retarding the flame, to reduce the risk of fire, either by providing resistance to ignition or by reducing the rate of combustion and thus delaying the spread of the flame.

During the Life-FLAREX project [1], different flame retardant products, currently on the market, have been studied: Halogenated FR and different FR alternatives. The technical feasibility and required flame retardant properties of alternatives and halogenated compounds was analyzed in 4 final applications such as bed sheets, mattresses ticking, upholstery and curtains.

\section{Discussion}

There are FR alternatives based on phosphonitrogen compounds and based on sulphonated nitrogenous, viable for textile applications that do not require fastness to washing (mattress ticking) as well as phosphonitrogenous alternatives for applications that require high durability to washing and whose fabrics contain cotton where the FR can be permanently bonded (bed sheets). Phosphonitrogenated alternatives have been found for textiles that require some wash fastness (curtains). An alternative based on expandable graphite has been tested for applications in upholstery fabrics that require resistance to fabric wetting. 
Some home textiles, such as bedclothes or mattress ticking, are in contact with skin for long periods, while people sleeping. In the present study, a step of LIFE-FLAREX project had the aim focused on dermal absorption of FR. Until now, no studies were found about the textile release of FR to the skin in contact with them. The growing evidence that suggests dermal absorption to be potentially significant pathway of human exposure to FRs, supports the interest in the study of percutaneous absorption of the FR $[2,3]$.

The dermal toxic potential of compounds applied to cotton has been studied, using the percutaneous absorption technique in
Franz cells according to the OECD methodology [4].

The absorption percutaneous experiments have been done with cotton and polyester fabrics containing flame retardants based on as phosphonitrogenous compounds, in concrete, sulfamate ammonium, with the aim of determine the amount of active substance that may cross the stratum corneum and to enter into deeper skin layers, following the experimental methodology described in previous studies $[5,6]$. In Figure 1 there is a scheme of percutaneous absorption process.
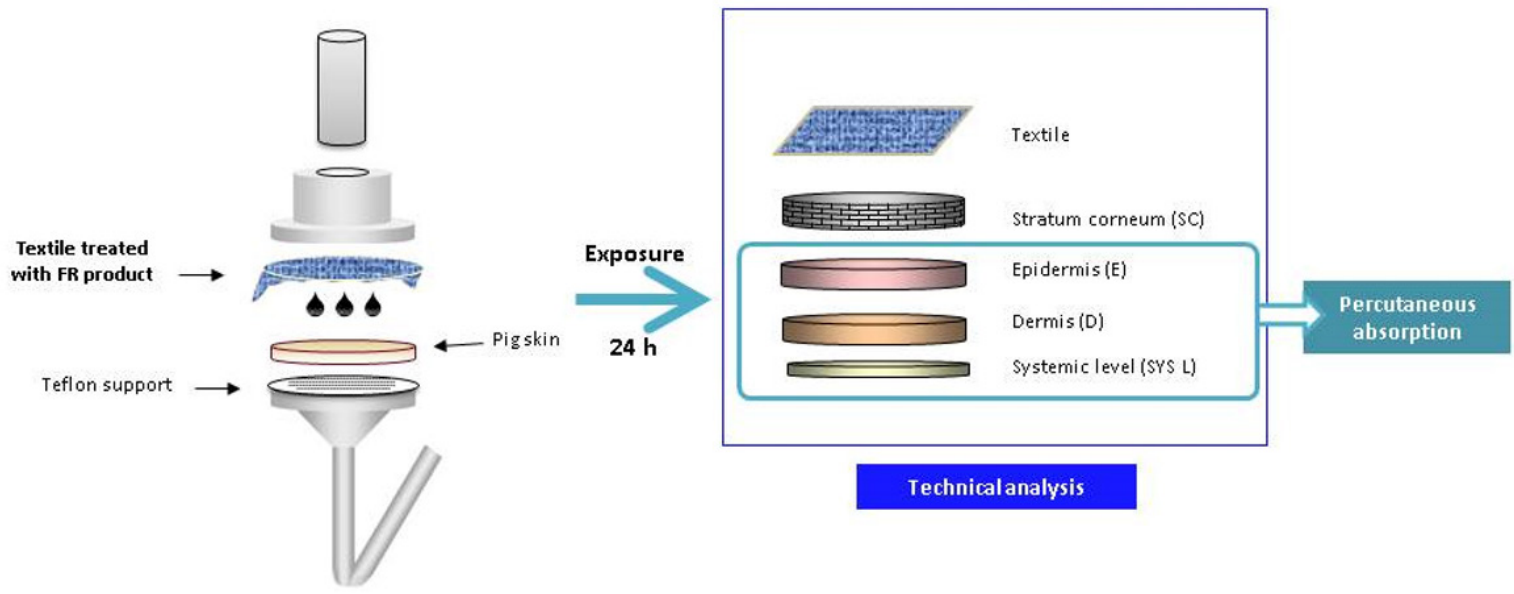

Figure 1: Percutaneous absorption scheme.

After percutaneous penetration was obtained, the value is used to calculate the MoS, margin of security of each FR compound studied [5].

$$
M o S=\frac{\text { NOAEL }}{S E D}
$$

Where NOAEL is the critical non-observed adverse effect level and SED is the systemic exposure dose.

In the next table there are the results obtained. In the ammonium sulphamate case the NOAEL is $1000 \mathrm{mg}$ compound $/ \mathrm{kg}$ body weight (bw)/day.

FR finished fabrics with MoS greater than 100 are safe for human health, so in this study, Table 1 both FR applied to a cotton bedding fabric and to polyester mattress ticking are not potentially toxic to humans in the quantities used to obtain a suitable flame retardant finish. Moreover, the FR alter seems to be more safety when in contact with the skin considering also its higher concentration in the textile.

Table 1: Values of the different parameters needed to calculate SED and the MoS value obtained.

\begin{tabular}{|c|c|c|c|c|c|c|}
\hline Application & $\begin{array}{l}\text { FR Active sub- } \\
\text { stance }\end{array}$ & $\begin{array}{l}\text { Specific fabric } \\
\text { composition }\end{array}$ & Dermal Absorption $\left(\mu \mathrm{g} \mathrm{cm}^{2}\right)$ & Skin Surface area & Freq. (daily ${ }^{-1}$ ) & $\begin{array}{l}\text { Margin of } \\
\text { Safety }\end{array}$ \\
\hline Mattress ticking & $\begin{array}{l}\text { Ammonium sul- } \\
\text { phamate }\end{array}$ & $\begin{array}{l}100 \% \text { PES } \\
(13 \% \text { owf })\end{array}$ & $\begin{array}{l}\text { b.l.d. } \\
<1,102\end{array}$ & Total body area & 3.5 & $\begin{array}{l}\text { SAFETY } \\
>900\end{array}$ \\
\hline Bedding sheets & & $\begin{array}{c}100 \% \text { CO } \\
\text { (79\% owf) }\end{array}$ & $\begin{array}{c}\text { b.l.d } \\
<1,102\end{array}$ & Total body area & 3.5 & $\begin{array}{l}\text { SAFETY } \\
>900\end{array}$ \\
\hline
\end{tabular}

\section{Conclusions}

In the study of the dermal toxic potential of FR-treated cotton bed sheets and polyester mattress ticking, it has been shown that neither none of the two products tested penetrate into the skin is toxic when applied under the conditions used. Therefore, even the more concentrate alternative FR, they all are safe when in contact with the skin.

\section{Acknowledgement}

Authors wish to thank the LIFE program from the European Union for its financial contribution to the LIFE-FLAREX (LIFE16 ENV/ES/000374) project.

\section{Conflict of Interest}

Authors declare no conflict of interest. 


\section{References}

1. (2019) LIFE-FLAREX project.

2. Rovira J, Domingo JL (2019) Human health risks due to exposure to inorganic and organic chemicals from textiles: A review. Environ Res 168: 62-69.

3. Frederiksen M, Vorkamp K, Jensen NM, Sorensen JA, Knudsen LE, et al. (2016) Dermal uptake and percutaneous penetration of ten flame retardants in a human skin ex vivo model. Chemosphere 162: 308-314.
4. (2004) Guideline for the testing of chemicals. Skin absorption: in vitro method. OECD. Organisation for Economic Cooperation and Development: 428.

5. Martí M, Alonso C, Manich AM, Coderch L (2020) Thermal Analysis (DSC, TGA) of textile flame retardants with less environmental impact. Rev De Química e Ind Textil 233: 11-21.

6. Martí M, Martínez V, Carreras N, Alonso C, Lis MJ, et al. (2014) Textiles with gallic acid microspheres: in vitro release characteristics. J Microencapsul 31(6): 535-541. 